\title{
Ciudadanía biológica: la ciencia y la política de las poblaciones expuestas a Chernóbil
}

\section{Adriana Petryna*}

\section{Resumen}

En la transición del socialismo al capitalismo de mercado, los cuerpos, las poblaciones y las categorías de ciudadanía han sido reordenados. La gestión racional y técnica de los grupos afectados por el desastre de Chernóbil en Ucrania es una ventana a este controvertido proceso. Chernóbil ejemplifica un momento en el que el conocimiento científico se colapsó y emergieron nuevos mapas y categorías. Los modelos antiguos de bienestar dependen de definiciones precisas que sitúan a los ciudadanos y sus atributos en un embrollo cruzado de categorías conocidas en las que se basan las reclamaciones de derechos. En ello se observa cómo las ambigüedades relacionadas con la categorización del sufrimiento crearon un campo político en el que un estado, formas de ciudadanía y economías informales fueron reconstruidos.

\section{Palabras clave}

Ciudadanía biológica; ciencia; etnografía; cuerpos; Chernóbil.

\section{TitLe}

Biological Citizenship: The Science and Politics of Chernobyl-Exposed Populations

\section{Abstract}

In the transition out of socialism to market capitalism, bodies, populations, and categories of citizenship have been reordered. The rational-technical management of groups affected by the Chernobyl disaster in Ukraine is a window into this contested process. Chernobyl exemplifies a moment when scientific knowability collapsed and new maps and categories of entitlement emerged. Older models of welfare rely on precise definitions situating citizens and their attributes on a cross-mesh of known categories upon which claims rights are based. Here one observes how ambiguities related to categorizing suffering created a political field in which a state, forms of citizenship, and informal economies were remade.

\section{KEYWORDS}

Biological citizenship; science; ethnography; bodies; Chernobyl.

\section{*Adriana PETRYNA,} Profesora de Antropología de la Universidad de Pensilvania, ocupando la plaza Edmund J. y Louise W. Kahn. Su dirección de correo electrónico es petryna@sas.upenn. edu.

Este artículo ha sido traducido con el permiso de la editorial University of Chicago Press, a cargo de la revista académica Osiris, así como de su autora Adriana Petryna. Publicado originalmente como PETRYNA, Adriana, "Biological Citizenship: The Science and Politics of ChernobylExposed Populations" en OSIRIS, n० 19, pp. 250-265.

Traducido por Elisa LÓPEZ, Magíster en Cooperación Internacional para el Desarrollo por la Universidad Complutense de Madrid.

DOI: $10.15366 /$ relacionesinternacio nales2017.35.005 
"El sentido común es lo que queda cuando toda clase de sistemas simbólicos articulados han agotado sus deberes". Clifford Geertz, Local Knowledge ${ }^{1}$

\section{ntroducción}

Este ensayo explora las formas de cooperación científica y gestión política que emergieron después del desastre nuclear de Chernóbil en 1986. Trata de cómo dichas gestiones están interconectadas con los flujos globales de tecnología y su integración en los procesos de construcción del estado, las nuevas estrategias de mercado y la gobernanza y ciudadanía en la Ucrania pos-soviética. Junto con estas dinámicas, el ensayo considera, a través de un ejemplo etnográfico, cómo las reivindicaciones locales de enfermedad y salud son refractadas a través de dichas instituciones, cómo los contextos sociopolíticos en los que el conocimiento científico se construye pueden influir en el curso particular de salud, enfermedad y las soluciones a estas condiciones. El objetivo aquí es articular las circunstancias a través de las cuales llegan a existir comunidades de población "en riesgo"; mostrar cómo las normas de ciudadanía tienen relación con dichas circunstancias; y cómo dichas normas se propagan a través de los conocimientos y prácticas científicas diarias relacionadas con instituciones de la medicina y la ley en Ucrania. Un conjunto de relaciones laborales se forma o está en juego en la propagación de las afirmaciones individuales de estar en riesgo. Incluyen las ciencias de instituciones globales y los expertos, las ciencias y leyes nacionales, las contingencias burocráticas locales, y las dinámicas familiares del sufrimiento. Estas relaciones están, ciertamente, "funcionando" en el sentido en que afectan a las percepciones de la severidad y la escala del desastre, las afirmaciones de su daño continuado, y la forma científica, económica y política de abordar dicho daño. ¿Cómo los diferentes sistemas de modelar el riesgo de Chernóbil afectan las capacidades de la gente para razonar políticamente?, ¿cómo podría la elección de enfermedades, antes que de salud, convertirse en una forma de "sentido común" expresivo de dichos modelos? Estas preguntas se exploran en un contexto en el que la ciencia está irremediablemente conectada a los procesos de construcción del estado, y los desarrollos del mercado están interconectados de forma bastante prolífica, generando nuevas instituciones y órdenes sociales a través de las cuales la ciudadanía, la experiencia y la ética están siendo alteradas.

Mi libro, Life Exposed: Biological Citizens after Chernobyl, dilucida cómo se utilizó el conocimiento científico y el sufrimiento que causó Chernóbil para lograr la equidad social en la dura transición a una economía de mercado. Más ampliamente, mostraba que en este nuevo estado, la ciencia y la política se vieron inmersas en un proceso constante de intercambio y estabilización mutua ${ }^{2}$. Este ensayo se construye sobre ese material, mostrando cómo los discutidos intentos de intervenir y cuantificar el riesgo de radiación formaron la naturaleza del pos-dañado régimen legal y político. Vista longitudinalmente, la catástrofe de Chernóbil ejemplifica un proceso en el que se colapsa el conocimiento científico y surgen nuevas categorías de derecho. Las ambigüedades relacionadas con la categorización del sufrimiento crean un campo político en el que el estado, formas de ciudadanía y economías informales

1 GEERTZ, Clifford, Local Knowledge: Further Essays in Interpretive Anthropology, Basic Books, Nueva York, 1983, p. 92.

2 PETRYNA, Adriana, Life Exposed: Biological Citizens After Chernobyl, Princeton University Press, Princeton, 2002. 
de la sanidad y los derechos son construidos nuevamente. Esta apropiación del sufrimiento a todos los niveles es un aspecto de cómo las imágenes del sufrimiento se materializan cada vez más en sus dimensiones legal, económica y política ${ }^{3}$. Este ensayo se preocupa especialmente por cómo estas materializaciones se convierten en una forma de sentido común y son representadas por los sufridores pudiendo intensificar los riesgos políticos del sufrimiento y promover la protección, así como nuevos tipos de vulnerabilidad en las áreas doméstica, científica y burocrática.

\section{El evento}

El reactor nuclear Unidad Cuatro de Chernóbil explotó en Ucrania el 26 de abril de 1986. Los daños de este desastre han sido diversos, incluyendo heridas inmediatas en forma de quemaduras por radiación, fallecimiento de los trabajadores de la planta, sistemas inmunitarios dañados, altas tasas de cáncer de tiroides entre las poblaciones reasentadas, y sustancial contaminación de campos y ríos. Los informes soviéticos atribuyeron la causa del desastre a un experimento fallido. Según un informe oficial: "El propósito del experimento era probar la posibilidad de usar la energía mecánica del rotor en un turbo-generador desconectado del suministro principal para mantener la cantidad de energía requerida durante un fallo energético"4. Muchos de los sistemas de seguridad del reactor se apagaron durante la duración del experimento. Se produjo una enorme sobrecarga eléctrica cuando los técnicos disminuyeron la potencia y cortaron el suministro principal. La Unidad explotó una vez a la 1:23 a.m. y después otra vez. Debido a gradientes particulares de la presión del viento ese día y en las semanas posteriores, la nube radioactiva se movió a una altura estimada de ocho kilómetros. Los sucesivos intentos de extinguir las llamas del núcleo de grafito sólo tuvieron éxito en parte. Para la mayoría, incluso exacerbaron el peligro de la situación. Por ejemplo, se intentó sofocar las llamas con toneladas de carburo de boro, dolomita, arena, barro y plomo lanzados desde helicópteros. Como resultado, la temperatura del núcleo aumentó. La nube de radiación aumentó dramáticamente y se movió a lo largo de Bielorrusia, Ucrania, Rusia, Europa del Este y otras áreas del Hemisferio Norte ${ }^{5}$.

Casi tres semanas después del evento, llegaba un anuncio oficial del desastre. En ese tiempo, casi 13.000 niños en áreas contaminadas estuvieron expuestos a dosis de radiación en la tiroides dos veces superiores a la más alta permitida para los trabajadores de la planta en un año ${ }^{6}$. Una aparición masiva de cánceres de tiroides en adultos y niños comenzó cuatro años más tarde. Si se hubieran puesto a disposición de la población píldoras de yodo no radioactivo en la primera semana del desastre, los casos de esta enfermedad se podrían haber reducido significativamente. Los administradores soviéticos contradijeron las estimaciones de grupos de meteorólogos ingleses y americanos sobre la escala de la nube. Los soviéticos afirmaron que los aspectos biomédicos de Chernóbil estaban bajo control. La doctora Angelina Guskova del Instituto de Biofísica de Moscú seleccionó inicialmente 237 víctimas para ser trasladadas

\footnotetext{
3 KLEINMAN, Arthur y KLEINMAN, Joan, "The Appeal of Experience; The Dismay of Images: Cultural Appropriations of Suffering in Our Times" en Daedalus, vol. 125, no 1, 1999, pp. 1-24. Ver también DAS, Veena, Critical Events: An Anthropological Perspective on Contemporary India, Oxford University Press, Nueva Deli, 1995.

4 Ver Comité Soviético para la Utilización de la Energía Atómica, Informe para la IAEA, Viena, 1986, p. 16

$5 \mathrm{SICH}$, Alexander, "The Denial Syndrome (Efforts to Smother the Burning Nuclear Core at the Chernobyl Power Plant in 1986 Were Insufficient)" en Bulletin of Atomic Scientists, vol. 52, 1996, pp. 38-40.

6 SHCHERBAK, Yurii, "Ten Years of the Chernobyl Era" en Scientific American, Abril, 1996, p. 46.
} 
a la sala de enfermedades por radiación aguda del Instituto. El síndrome de irradiación aguda (SIA) fue diagnosticado a 134 de ellos. La tasa oficial de muertos fue de 31 personas, la mayoría bomberos y trabajadores de la planta.

El desastre continuó, especialmente entre los grupos de trabajadores que fueron reclutados como voluntarios para trabajar en el lugar del desastre. Para los cientos de miles de trabajadores remunerados 0 no $^{7}$, los trabajos iban desde nivelar el suelo contaminado y desechar los residuos en los contenedores para radiación (mohyl'nyky), retirar piezas del núcleo del reactor -grafito radiactivo- que se habían dispersado por una vasta área, construir vayas alrededor del reactor, hasta talar los bosques de los alrededores altamente contaminados. El trabajo más peligroso con diferencia incluía el tejado adyacente al reactor. En intervalos de un minuto, los trabajadores -en su mayoría reclutas militares - corrían al tejado, arrojaban los escombros radiactivos de los parapetos en los contenedores de debajo con sus palas y después se iban. Muchos de estos voluntarios se llamaban a sí mismos "biorobots"; sus biologías fueron explotadas "y luego desechadas". Según extensas entrevistas, algunos trabajadores se sintieron atrapados e incapaces de dejar el área del desastre; este sentimiento lo tenían principalmente los reclutas militares no remunerados y los colectivos locales de granjeros reclutados para hacer las tareas más difíciles y peligrosas. Algunos dicen que fueron con gusto creyendo que recibirían un salario que compensaría el riesgo. Sin embargo, definitivamente no puede decirse que el dinero les compensara verdaderamente por el sufrimiento que estaba por venir.

Cinco meses después del desastre, un entonces llamado sarcófago - ahora simplemente Ilamado El Asentamiento - se construiría para contener las 216 toneladas de uranio y plutonio en el reactor en ruinas. En el presente, la planta eléctrica ha sido desmantelada. Unas quince mil personas llevaron a cabo los trabajos y servicios de mantenimiento de la Zona de Exclusión. La mayor parte de la Zona de Exclusión se encuentra en Ucrania. La zona circunscribe el lugar del desastre y cubre treinta kilómetros a la redonda. La entrada a la zona está limitada a los trabajadores de la planta.

Ucrania heredó la planta energética y la mayor parte de la Zona de Exclusión cuando declaró su independencia en 1991. El gobierno anunció nuevos y ambiciosos estándares de seguridad. Focalizó sus recursos en estabilizar el ruinoso asentamiento, implementando normas de seguridad para los trabajadores; reduciendo el riesgo de futuras fugas y desmantelando todas las unidades de la planta de Chernóbil. Estos actos fueron importantes desde una perspectiva de política exterior. Mostrando que podía adherirse a estrictos estándares de seguridad, Ucrania se convirtió en el receptor de la ayuda técnica, los préstamos y las asociaciones comerciales europeas y americanas. El legado de Chernóbil se ha utilizado como un medio para señalar la legitimación interna e internacional de Ucrania y reafirmar sus reclamaciones territoriales; y como un espacio de gobernanza y construcción de estado, bienestar social y corrupción.

Algunos trabajadores de mantenimiento vivían en unidades de alojamiento construidas

\footnotetext{
7 Las estimaciones varían desde 600000 a 800 000. Estos trabajadores venían de todo el territorio de la Unión Soviética. El grupo de trabajo, sin embargo, se veía más entre poblaciones rusas y ucranianas.
} 
por el Gobierno en Kiev, la capital del país, sesenta millas al sur de la zona del desastre. Trabajaban en la zona dos semanas y luego regresaban a casa otras dos semanas. Conocí a uno de estos trabajadores en 1992, la primera vez que viajé al país. Se identificó a sí mismo como un "sufridor", calificación legal instituida en 1991 para los individuos afectados por Chernóbil. Se quejó sobre lo insignificante que era la compensación -alrededor de unos cinco dólares americanos al mes- en relación al aumento del precio de los alimentos ${ }^{8}$. El hombre estaba totalmente desesperado, atrapado por no tener ningún sitio más donde trabajar. Dijo que había tratado de encontrar empleo en otros sitios pero nadie quería contratarle por su mala salud e historial de trabajo. El hombre asociaba su sufrimiento a la precaria y peligrosa gestión soviética inicial del desastre, y luego a un complejo aparato médico y legal en el que se sintió incapaz de manejarse. Después me enseñó una herida de trabajo, un trozo de piel que se había podrido y formado una especie de anillo justo sobre el tobillo. Aparentemente causado por el contacto directo con una fuente de radiación iónica. Su sentimiento de violación y pérdida eran evidentes cuando se refería a sí mismo como un "muerto viviente" cuya memoria de quién había sido en una vida anterior "había desaparecido".

En el año 2000, entrevisté al director del complejo de reasentamiento. Lo que aprendí es que casi una década después de la independencia, las protecciones de los trabajadores, en lugar de mejorar, eran todavía deficientes. El director me dijo que las normas de seguridad por radiación estaban inoperativas. En un momento de tremenda depreciación económica, la gente competía por trabajar en la Zona de Exclusión, donde los salarios eran relativamente altos y estables. Los potenciales trabajadores hicieron la siguiente estimación problemática de coste-beneficio: si trabajo en la Zona, pierdo mi salud. Pero puedo mandar a mi hijo a la facultad de derecho. "Correr este riesgo es su problema individual. Nadie más es responsable de ello" me dijo el director. Comparó el modo en que Ucrania reforzó los estándares de seguridad con el europeo y me dijo que el "valor" de una dosis de exposición se mantuvo incalculable en Ucrania. En Europa, dichos valores se calculan en base a los gastos en los que incurren los trabajadores; los estándares de seguridad internacionales limitan las cantidades. A pesar de la existencia de dichas limitaciones internacionales, el comentario del director sugiere que las normas de exposición de los trabajadores se deciden localmente y dentro de las limitaciones de una economía local. En efecto, me estaba revelando hasta qué punto las vidas de los trabajadores son infravaloradas al ser sobre expuestos - por mucho menos salario-. Sin embargo, aunque las vidas de sus trabajadores fueran infravaloradas, son empujados al trabajo por verse forzados ante una situación en la que las fuerzas económicas son abrumadoras. En este entorno, la escalada del riesgo físico y el trabajo arriesgado se ve como aceptable e incluso normal.

"Como resultado de todos los componentes inciertos en los factores involucrados," escribió Frank von Hippel, "nuestras estimaciones de las consecuencias en la salud a largo plazo del accidente de Chernóbil son inciertas incluso en su magnitud" ${ }^{\prime \prime}$. De hecho, los modelos

\footnotetext{
8 El karbóvanet (Krb) era la moneda legal en Ucrania de 1992 a 1996. Las tasas de cambio relacionadas a 1 USD, se desmoronaron entre 1992 y 1993. En marzo de 1992, la tasa de cambio era 64 Krb por 1 USD. En marzo de 1993, había caído a $12.610 \mathrm{Krb}$ por 1 USD. Las tasas subsecuentes fueron como sigue: 1994- $14.200 \mathrm{Krb}$ a 1 USD; 1995- 179.900 Krb a 1 USD; 1996- 188.700 Krb a 1 USD. La grivna (Hm) reemplazó el karbóvanet a $1 \mathrm{Hm}$ por $1.000 \mathrm{Krb}$ en septiembre de 1996. Las tasas de cambio siguieron así: $1997-1,84 \mathrm{Hm}$ a 1 USD; 1998- 2,04 Hm a 1 USD; 1999- 4,13 Hm a 1 USD; 2000- 5,44 Hm a 1 USD.

9 HIPPEL, Frank von, Citizen Scientist, The American Institute of Physics, Nueva York, 1991, p. 235.
} 
disponibles de estimación no podrían dar cuenta del alcance del desastre. Como indica la corta historia del desastre, las respuestas técnico-racionales y las administraciones políticas -en ambos periodos soviético y ucraniano- han sido factores complejos en la tragedia médica y de bienestar que ahora afecta a más de 3,5 millones de personas sólo en Ucrania. Discutidos descubrimientos científicos sobre la extensión y el impacto médico del desastre, la decisión de posponer las comunicaciones públicas y el ímpetu económico de trabajar en la Zona de Exclusión han hecho de Chernóbil una Tekhnohenna katastrofa - una catástrofe tecnogénica-. Este es un término usado por mis informantes -incluyendo gente que luchaba por el estatus de discapacidad-, físicos locales y científicos. Esto sugiere que no sólo la exposición a la radiación, sino también la gestión política ha producido nuevas incertidumbres biológicas.

Ulrich Beck se dio cuenta de que Chernóbil supuso un "shock antropológico" en Europa del Este. El shock se debió al hecho de que el conocimiento cotidiano demostró ser insuficiente para afrontar esta catástrofe, como lo fue el conocimiento experto ${ }^{10}$. Este "colapso" del conocimiento ocurrió también, pero de otra manera, en la otra Europa. Chernóbil se asoció al colapso de la vida soviética en general. El conocimiento sobre el riesgo, cómo actuar, cómo valorarlo se convirtió en algo así como un recurso político. En este desastre se despertó un estado, una sociedad y el conocimiento y la experiencia sobre la salud fueron reconfigurados.

Para explorar esta catástrofe, utilizo un enfoque metodológico que incluye el movimiento hacia adelante y atrás entre personas vulnerables, burocracias y procedimientos cotidianos, a través de los cuales expresan sus deseos, reclamaciones y necesidades de protección y seguridad. Un compromiso tan etnográfico está destinado en sí mismo a cuestionar la posibilidad de una explicación lineal o una solución política y moral sin medias tintas para esta compleja realidad. De hecho, sus dinámicas se estudian desde un punto de vista prismático para ganar una perspectiva más amplia de los intereses y valores envueltos en afirmaciones y lugares particulares.

\section{Modelos experimentales y métodos etnográficos}

Entre 1992 y 1997, llevé a cabo una investigación de archivo y de campo en Ucrania, Rusia y Estados Unidos. En Ucrania, trabajé con familias reasentadas y trabajadores expuestos a la radiación. También investigué los archivos del nuevo Ministerio de Chernóbil en este país, del Ministerio de Salud y de la Comisión Parlamentaria de los Derechos Humanos. Entrevisté a científicos y actores políticos clave en Kiev y Moscú, comparando los estándares científicos de los conceptos informativos de riesgo biológico y seguridad en las administraciones soviéticas y pos-soviéticas de la catástrofe. La naturaleza del problema, es decir, entender los aspectos del desastre de Chernóbil en la vida cotidiana, me llevó a numerosos lugares y retos. Uno de esos retos incluía entender cómo el conocimiento científico sobre el riesgo de la radiación se divulgaba, era asimilado o rechazado en los distintos niveles -internacional, nacional y local- en los que se estaban realizando intervenciones. Examiné las afirmaciones hechas sobre la escala del desastre por científicos expertos afiliados a la Agencia Internacional de Energía Atómica. Comparé el conocimiento experto con el de científicos de laboratorios

\footnotetext{
${ }^{10}$ BECK, Ulrich, "The Anthropological Shock: Chernobyl and the Contours of a Risk Society" en Berkeley Journal of Sociology, vol. 32, 1987, pp. 153-165.
} 
americanos de radiación y me enteré de cómo los radiobiólogos se dedicaban a evaluar los efectos radiobiológicos a nivel celular y subcelular.

Como consecuencia, pude situar mejor las afirmaciones y mediciones de los expertos en el contexto de sus producciones y pruebas de laboratorio. Pronto descubrí que había una "caja negra" que separaba el conocimiento sobre los efectos de bajas dosis de radiación en animales - laboratorio- y en humanos - campo-. Las curvas de efecto de las dosis para altas dosis de radiación eran unívocas y bastante claras. No se puede decir lo mismo de exposiciones regulares a bajas dosis - una condición típica tras Chernóbil-. Por un lado, los expertos promovían su autoridad, basada en parte en su control sobre qué se consideraba evidencia de una herida relacionada con Chernóbil. Por otro, había un considerable desacuerdo a nivel de laboratorio sobre cuáles eran los términos para interpretar el riesgo biológico inducido por radiación en poblaciones humanas. Las proyecciones de los expertos internacionales sobre los efectos de Chernóbil en la salud a menudo contradecían los efectos vividos por la gente. Para científicos ucranianos, la falta de consenso a un nivel científico tan básico significaba que los criterios de evaluación de las heridas eran, en esencia, discutibles.

Ucrania se convirtió en el lugar más pertinente para examinar las relaciones entre el riesgo, el poder técnico-racional, y el surgimiento de nuevas poblaciones. De hecho, apareció un nuevo escenario político, económico y moral gracias a la ausencia de criterios de evaluación consistentes. Durante el periodo de mi investigación de campo, el país vivió el crecimiento de una población que reivindicaba que la exposición a la radiación les calificara para alguna forma de protección social. La protección social incluía subsidios en efectivo, subsidios familiares, servicio sanitario y educación gratuitos y pensiones para los sufridores y discapacitados. Esta nueva población, llamada poterpili - sufridores-, unos 3,5 millones, constituía un $7 \%$ de la población. Estaba surgiendo una economía política de enfermedades relacionadas con Chernóbil con nuevos tipos de categorías sociales y jerarquías de derechos. Un individuo clasificado como "discapacitado" recibía mejor paquete compensatorio que un mero "sufridor". Los no sufridores -es decir, el sistema de compensación para gente de fuera de Chernóbil-, tenían todavía menos o ninguna posibilidad de recibir ayudas sociales del estado. El conocimiento científico se convirtió en esencial para las negociaciones de la vida cotidiana y el mantenimiento del estatus en el sistema de Chernóbil. Uno tenía que conocer la dosis a la que había estado expuesto y ser capaz de relacionarla con los síntomas y con las experiencias de trabajo en la Zona de Exclusión. La efectividad de este conocimiento determinaba el lugar que uno ocupaba y por cuánto tiempo en el sistema de gestión de las poblaciones de Chernóbil.

Hoy, aproximadamente el 8,9\% de Ucrania se considera contaminada. De media, el $5 \%$ del presupuesto del país va para gastos relacionados con Chernóbil. Esto incluye costes relacionados con la limpieza del medio ambiente y el soporte técnico del reactor destruido. La mayoría de los fondos $-65 \%-$, sin embargo, se gastan en compensaciones sociales y el mantenimiento financiero del aparato científico y de salud pública de Chernóbil. Bielorusia fue afectada en mayor medida que Ucrania. Cerca del 23 por ciento de su territorio está contaminado. Sin embargo, Bielorusia gasta mucho menos que su vecino del sur en la población afectada; ha contenido la suma de demandantes de Chernóbil -al igual que 
Rusia- ${ }^{11}$. La doctora Guskova, que supervisó el sistema de compensación ruso para los trabajadores de las instalaciones nucleares, incluyendo Chernóbil, es una conocida crítica del sistema compensatorio ucraniano. Esta me dijo que los ucranianos estaban inflando sus números de personas expuestas, que sus llamados inválidos "no querían recuperarse". Veía las enfermedades de este grupo como una "lucha de poder y recursos materiales a consecuencia del desastre".

En respuesta a la acusación de su colega de profesión, la doctora Agelina Ceanu, neurofisióloga y física de las víctimas de Chernóbil en Kiev, me dijo, "es inconcebible que un organismo de ningún tipo sea pasivo a su propia destrucción". Su respuesta se basaba en la evidencias de experimentos llevados a cabo por el radiobiólogo soviético V.L. Komarov. En un experimento llevado a cabo a finales de los cincuenta, observó que las ratas dormidas, sin ser provocadas, se despertaban cuando eran expuestas a pequeñas cantidades de radiación ionizada. Con estos ejemplos uno puede empezar a apreciar cómo modelos científicos antagónicos -animal/humano, psicométrico/biológico, laboratorio/trabajo de campo-, agendas de financiación, y actitudes morales respecto a la necesidad del trabajo científico en esta área no estaban especialmente en desacuerdo. Su confrontación abrió un nuevo escenario social de reclamaciones discutidas por enfermedades de radiación. De hecho, algunas organizaciones civiles presionaron por el derecho a una compensación de las instituciones biomédicas y políticas ucranianas que promovían la "vida segura" en Ucrania por dichas enfermedades. Estos llamados fondy - fondos - eran canales de caridad internacional y representaban la preocupación por la exclusión de los trabajadores de la Zona de Exclusión y personas reasentadas viviendo en Ucrania. Estos fondos disfrutaban de exención fiscal y con sus números -más de 500 en 1996- establecieron una economía informal de una gran variedad de productos importados, incluyendo vehículos, medicamentos, y productos alimenticios secos y congelados. En resumen, el desastre de Chernóbil se convirtió en un prisma de las problemáticas circunstancias políticas, económicas y sociales que tipificó la transición ucraniana a una economía de mercado. La producción de conocimiento científico y la formación de mercado y estado se integraron mutuamente generando nuevas desigualdades y oportunidades en la redefinición de la ciudadanía y la ética.

Este trabajo se basa en múltiples y largas visitas a varios contextos de estado, científicos y domésticos entre 1992 y 1995, el trabajo de campo llevado a cabo entre 1996 y 1997 y una visita de seguimiento en 2000. El Centro de Investigación de la Radiación, también conocido como Klinika, se convirtió en el primer foco de la investigación de campo. El Centro se estableció en 1986 para monitorear la salud de los trabajadores de la zona; poco después comenzó a proporcionar servicios similares a las personas reasentadas. El Comité Médico-Laboral a nivel nacional (Ekspertiza) reunía a científicos, físicos y administradores con autoridad para diagnosticar las enfermedades relacionadas con Chernóbil -hay doce comités regionales-. Los pacientes con enfermedades diagnosticadas como tal reciben un documento, el Ilamado Chernobyl tie, que otorga al paciente el privilegio de recibir una compensación como resultado de su enfermedad relacionada con Chernóbil. Para 1996, el Centro se había convertido en un lugar de intensas disputas legales y científicas. Observé

${ }^{11}$ En Rusia, el número de personas que se consideraron afectadas y sujetas a compensación se mantuvo en el mínimo y se mantiene bastante estable - unos 350.000, incluyendo 300.000 trabajadores de la Zona de Exclusión y 50.000 reasentados-. 
a médicos, enfermeras, y pacientes mientras negociaban quién debía recibir el documento. Busqué en investigaciones del momento, particularmente en la división neurológica del Centro. También hice entrevistas a sesenta pacientes, hombres y mujeres de mediana edad, y revisé sus historias médicas, la progresión de su enfermedad y sus experiencias para intentar alcanzar el estatus de discapacitado. Un aspecto significativo de mi investigación se centró en la vida diaria de los pacientes masculinos y sus familias. Me preocupaba cómo su pertenencia a una economía política de enfermedad desplazaba las percepciones de sí mismos y sus roles de figura paternal y cabeza de familia. Rastreé experiencias cambiantes de lichnost, un modelo ruso-soviético de personalidad evidenciado por la ética laboral de las personas y su nivel de compromiso con un colectivo de trabajadores ${ }^{12}$, los efectos que dichos cambios tuvieron en la vida doméstica, y las técnicas que utilizaban los miembros de una familia para hacer que sus enfermedades contaran en la esfera técnico-racional en la que encaraban su futuro.

Estas preocupaciones antropológicas ilustran hasta qué punto las definiciones de salud y enfermedad se integran en las esferas política y económica y están casi siempre conectadas con dimensiones que van más allá del cuerpo inmediato, como las relaciones interpersonales y domésticas. Arthur Kleinman ha dilucidado el "curso social" de la enfermedad"13. Otros antropólogos, como Veena Das y Nancy Seheper-Hughes, se han preocupado de cómo las construcciones de salud evidencian discrepancias en el poder, la posición social y la desigualdad, particularmente las vividas por grupos e individuos marginales. Etnografías recientes de la ciencia han retratado cómo, cada vez más, tecnologías biomédicas juegan un papel clave en estos paradigmas. Los escáneres PET, los diagnósticos basados en la genética y las máquinas de ultrasonido captan hechos biológicos y no se pueden, por tanto, separar de los objetos que reconocen y clasifican como enfermedad ${ }^{14}$. Los problemas sociales, de salud y las tecnologías que los captan también están relacionados. El antropólogo Paul Farmer ha mostrado cómo los patrones de "violencia estructural" afectan a la construcción y expansión de poblaciones en riesgo por enfermedades. El deterioro del sistema sanitario, la limitación de los tratamientos y las desigualdades han empeorado por los programas de ajuste estructural y han llevado a epidemias de enfermedades infecciosas evitables como la tuberculosis multirresistente. De hecho, "las fuerzas y procesos sociales se han convertido en eventos biológicos"15. En Ucrania, los esfuerzos para remediar los efectos de Chernóbil en la salud han contribuido a la indeterminación social y biológica y a nuevas formaciones de poder. La exposición a la radiación y su incomprensión, las intervenciones burocráticas del estado y los fracasos de las mismas, el crecimiento de los regímenes clínicos y los duros cambios del mercado intensificaron el curso de la enfermedad y el sufrimiento. Por consiguiente, en el desastre de Chernóbil, enfermedad y salud son engendradas y tienen sentido dentro de la esfera técnica y política en la que son abordadas.

12 KHARKHORDIN, Oleg, The Collective and the Individual in Russia: A Study of Practices, University of California Press, Berkeley, 1999.

${ }^{13}$ KLEINMAN, Arthur, Social Origins of Distress and Disease, Yale University Press, New Haven, 1986.

${ }^{14}$ MARTIN, Emily, Flexible Bodies: Tracking Immunity in American Culture from the Days of Polio to the Age of AIDS, Beacon Press, Boston, 1994; RAPP, Rayna, Testing Women, Testing the Fetus: The Social Impact of Amniocentesis on America, Routledge, Nueva York, 1999; DUMIT, Joseph, Picturing Personhood: Brain Scans and Biomedical Identity, Princeton University Press, Princeton, 2004.

${ }^{15}$ FARMER, Paul, Infections and Inequalities: The Modern Plagues, University of California Press, Berkeley, 1999, p. 5. 


\section{Desconocidos construidos}

En lo que sigue, abordo algunos de los elementos científicos que jugaron un papel clave en la medición y delimitación del alcance de la catástrofe y la definición de las estrategias de reparación y compensación. En este contexto, asuntos como los mapas de dispersión atmosférica, la cooperación científica internacional, y las respuestas científicas locales, así como el involucramiento de la gente en los procesos burocráticos y las pruebas, llevaron a lo que puede llamarse un "curso de la enfermedad técnico y político". Daré más adelante ejemplos de la implicación e influencia de la gente en estos cursos.

La mayoría de científicos hoy en día coincidirían que dado el estado de la tecnología en el momento del desastre, los especialistas "no sabían cómo hacer un diagnóstico objetivo de lo que había pasado"16. Tom Sullivan, quien hasta hace poco dirigía el grupo ARAC Atmospheric Release Advisory Capability- del Laboratorio Lawrence Livermore en Livermore, California, coincide con esta valoración general ${ }^{17}$. Antes del desastre de Chernóbil, el equipo de Sullivan en el ARAC había generado modelos de dispersión atmosférica sobre el tamaño y movimiento de nubes nucleares resultantes de las pruebas con armas nucleares americanas y chinas y el accidente de Three Mile Island. "Un área de 200 kilómetros cuadrados había sido suficiente para modelar anteriores filtraciones de radiación", me contó.

"Hicimos un escaneo cerca de la planta de Chernóbil usando esta cuadrícula de 200 kilómetros cuadrados, pero estaba demasiado saturada, es decir, ni siquiera tenía sentido porque todos los lugares tenían unos valores de radiación extremadamente altos [...]. Nuestros códigos no estaban preparados para un evento de tales magnitudes"18.

Los científicos soviéticos tampoco estaban preparados, pero no admitieron su ignorancia. En Agosto de 1986, la Agencia Internacional de Energía Atómica (IAEA), presentó un crudo análisis de la distribución de la radiación en la Zona de Exclusión y en la Unión Soviética: "se hicieron diagnósticos sobre las dosis de radiación presentes y futuras recibidas por la población de las ciudades, pueblos y otros lugares inhabitados. Como resultado de estas y otras medidas, se demostró que es posible mantener las exposiciones dentro de los límites establecidos"19.

Lo que está en juego es la capacidad del estado de producir y utilizar el conocimiento y desconocimiento científico para mantener el orden político. El historiador Loren Graham, por ejemplo, ha escrito sobre cómo las "falsas" ciencias como el Lysenkoisismo, que negó la existencia del gen y propuso métodos de trabajo intensivo para acelerar el rendimiento de las cosechas, han sido decisivas para dar forma a la psicología del trabajo y la vida social en el

${ }^{16}$ One Decade After Chernobyl, Viena, 1996.

${ }^{17}$ EI ARAC es un servicio nacional de respuesta a emergencias para una evaluación a tiempo real de incidentes con materiales peligrosos nucleares, químicos biológicos o naturales.

${ }^{18}$ El equipo de Sullivan ofreció asistencia técnica a través de un intermediario sueco, pero la oferta fue rechazada por los administradores soviéticos.

${ }^{19}$ SOVIET STATE COMMITTEE ON THE UTILIZATION OF ATOMIC ENERGY, The Accident at Cherobyl Nuclear Power Plant and Its Consequences, Información recogida en la Reunión de Expertos del IAEA, Ag. 25- 29, 1986, Viena; ZHORES Medvedev, The Legacy of Chernobyl, Nueva York, 1990. 
proyecto socialista ${ }^{20}$. El hecho es que los limitados mapas soviéticos de Chernóbil contribuyeron a justificar las también limitadas acciones de vigilancia y reconstrucción dosimétrica. El desconocimiento se convirtió en esencial para el despliegue del conocimiento acreditado. Las altas dosis absorbidas por al menos 200.000 trabajadores entre 1986 y 1987 fueron insuficientemente documentadas. De acuerdo con un bioquímico, muchos de los trabajadores que se ocuparon de la limpieza "recibieron de 6 a 8 veces más dosis de radiación que la considerada como letal"21. "Están vivos", me dijo. "Saben que no murieron. Pero no saben cómo sobrevivieron". Su declaración muestra hasta qué punto no sólo el conocimiento sino la ignorancia fue construida y utilizada como herramienta del estado para mantener el orden público. Como dijo el historiador científico Rober Proctor en su libro informativo sobre cómo la política influye en la ciencia del cáncer, la ignorancia "no es sólo una consecuencia natural de los límites, siempre cambiantes, entre lo conocido y lo desconocido", es una "consecuencia política" de las decisiones sobre cómo abordar lo que podría y debería hacerse para mitigar el daño o la enfermedad ${ }^{22}$.

Chernóbil se convirtió en un espacio de cooperación científica e investigación humana sin precedentes. El presidente Mikhail Gorbachev invitó personalmente a un equipo de oncólogos americanos liderado por el especialista en leucemia Robert Gale (UCLA) para llevar a cabo trasplantes experimentales de médula ósea en individuos cuya exposición estaba por debajo del límite letal y para los que el trasplante se consideraba apropiado. Además, 400 trabajadores seleccionados por la doctora Guskova y otros recibieron una molécula de factor de crecimiento hematopoyético genéticamente modificada (rhGM-CSF), pensada para regenerar el crecimiento de células madre. Aunque los resultados de los trasplantes y el experimento no tuvieron éxito, el trabajo médico en este aspecto $-\mathrm{y}$ los índices objetivos creados a raíz del mismo- ayudaron a consolidar una imagen de éxito en el control de la crisis biomédica a través de aplicaciones científicas rompedoras. En un esfuerzo por aliviar el temor de la población, el doctor Gale apareció en televisión y caminó descalzo por la zona con uno de sus hijos.

Contrariamente a esta internacionalización de la ciencia, la gestión física de la contaminación en el lugar del accidente fue internalizada - a la esfera de control soviético-. Una declaración del Ministerio de Sanidad Soviético en la cumbre de esta cooperación, por ejemplo, iba dirigida a los examinadores médicos en la Zona de Exclusión para que "clasificaran" a la enfermedad de los trabajadores que habían recibido una dosis máxima como "distonía vegetovascular", que es un tipo de desorden de ansiedad, y como un nuevo desorden psicosocial Ilamado "radiofobia" - o el miedo a la influencia biológica de la radiación-. Estas categorías se utilizaron para librarse de la mayoría de reclamaciones por discapacidad ${ }^{23}$. En

20 GRAHAM, Loren, What Have We Learned about Science and Technology from the Russian Experience?, Stanford University Press, Stanford, 1998.

${ }^{21}$ Los síntomas de enfermedad por radiación aguda empiezan con 200 rem. Con 400 rem aparecen los fallos de médula ósea. La dosis letal (LD100) es una dosis de exposición que causa la muerte del cien por cien de las células humanas. LD 50/30 es una dosis de exposición que causa la muerte del cincuenta por ciento de las células humanas en treinta días.

22 PROCTOR, Robert, Cancer Wars: How Politics Shapes What We Know and Don't Know about Cancer, Basic Books, Nueva York, 1995, p. 7.

${ }^{23}$ En mis entrevistas, escuché casos de trabajadores que imitaban síntomas del SIA - vómitos, por ejemplo-. Esto muestra la desesperación de algunos de ellos por recibir el permiso para dejar la Zona. 
los años siguientes esta gestión soviética tuvo que hacer frente a importantes desafíos de algunos sectores de trabajadores. Al final de 1989 sólo a 130 personas más se les garantizó la discapacidad; para 1990, 2.753 nuevos casos habían sido considerados, de los cuales el $50 \%$ fue autorizado por motivos neurológicos. Los niveles de influencia política de sectores específicos de trabajadores se reflejan en el orden en el que se les concedía la discapacidad: mineros del cobre, trabajadores del Ministerio de Interior - policías-, y después trabajadores del Ministerio de Transporte. Estos grupos de trabajadores se darían cuenta pronto que en la gestión ucraniana de Chernóbil, las influencias políticas tenían que ir acompañadas de razonamientos médico-científicos.

Se puede decir que el que Ucrania considerara lo desconocido en Chernóbil era parte de las estrategias del gobierno para una gobernanza y una movilización social basadas en el conocimiento. En 1991 en el primer conjunto de leyes, el nuevo parlamento denunció la gestión soviética de Chernóbil como "un acto de genocidio". El nuevo estado-nación vio el desastre como - entre otras cosas - un medio clave para instaurar su autoridad nacional e internacional. Los legisladores acometieron contra el estándar soviético para determinar el riesgo biológico de las poblaciones. Los soviéticos habían establecido una altura de 35 rem - unidad de dosis absorbida-, repartida a lo largo de la vida de un individuo -utilizando una media de 70 años-, como el umbral de dosis de radiación admisible. Esto limitó la escala de las acciones de reasentamiento. La ley ucraniana rebajó el umbral soviético a 7 rem, comparable a lo que un americano habría estado expuesto de media en toda su vida. En efecto, esta rebaja del límite para una vida saludable aumentó el tamaño de las fuerzas de trabajo en la Zona de Exclusión - los trabajadores tenían que trabajar menos tiempo si querían evitar exceder la dosis estándar-. Las medidas también expandieron los territorios considerados contaminados. Un significativo nuevo sector de la población quería proclamarse parte del sistema de protección pos soviético. Uno de los biofísicos responsables de conducir la evaluación de dosis retrospectivas en el reasentamiento me dijo:

"Largas filas de reasentados se extendían desde las puertas de nuestro laboratorio. No era suficiente que fueran evacuados a áreas 'limpias'. La gente quedó atrapada en la categoría de víctima, por ley. Tenían un futuro impredecible, y cada uno quería conocer su dosis".

Las estadísticas del Ministerio Ucraniano de Salud evidencian el agudo incremento en 1991 de trabajadores de la zona, personas reasentadas y habitantes de territorios contaminados que registraron su discapacidad, y de los patrones anuales de inscripción de esta nueva población que el estado se comprometió a cuidar. Las estadísticas también muestran que el mayor incremento de los registros clínicos de enfermedad fue bajo la categoría de "síntomas y otras condiciones insuficientemente conocidas", la Clase 16 de la Clasificación Internacional de Enfermedades CIE-10 - ver figura 1-. Estas condiciones incluían típicamente aflicciones como cambios de personalidad, senilidad prematura y psicosis.

Figura 1. Síntomas y Otras Condiciones Insuficientemente Conocidas - por 10.000-

\begin{tabular}{|r|r|r|r|r|r|r|r|r|r|r|}
\hline 1982 & 1983 & 1984 & 1985 & 1986 & 1987 & 1988 & 1989 & 1990 & 1991 & 1992 \\
\hline 1,3 & 1,7 & 1,7 & 1,9 & 2,3 & 2,7 & 5,9 & 34,7 & 108,3 & 127,4 & 141,3 \\
\hline
\end{tabular}

FUENTE: Ministerio de Estadística, Kiev, Ucrania. 
La declaración ucraniana de una repentina expansión de los efectos de Chernóbil en la salud fue recibida con escepticismo internacional. Se reprochaba a los científicos ucranianos el "no haber utilizado métodos epidemiológicos modernos, criterios de causalidad y sistemas fiables de datos". Como notificó un consultor del Banco Mundial, "ahora mismo prácticamente cualquier enfermedad es atribuida a Chernóbil y no se está haciendo ningún esfuerzo, que satisfaga los criterios epidemiológicos estándar de causalidad, por probar o refutar estas afirmaciones" ${ }^{\prime 24}$. Se puede decir que, para el gobierno, sin embargo, estas nuevas estadísticas se convirtieron en una especie de "ciencia moral", ${ }^{25}$ una decidida demostración de su intención de hacer visibles los efectos de la mala gestión soviética del desastre y garantizar su propia legitimación social mientras mantenía la atención del mundo en el riesgo de Chernóbil.

En esta ejemplificación burocrática cotidiana de Chernóbil, las tensiones entre trabajadores de la zona, individuos y familias reasentadas, científicos, físicos, legisladores y funcionarios, se intensificaron. Juntos, estos grupos se vieron envueltos en un nuevo contrato social y moral entre el estado y la sociedad civil, un contrato que les garantizaba el derecho a saber sus niveles de riesgo y a utilizar medios legales para obtener cuidado y seguimiento médico. A sufridores y administradores también los mantenían los ciudadanos no sufridores, quienes pagaban un impuesto del $12 \%$ de su salario para soportar las compensaciones. La calidad híbrida de este estado y contrato social pos socialista sale a la luz. Por un lado, el gobierno ucraniano rechazó las propuestas del oeste neoliberal de recortar el bienestar social; por otro lado, se presentó a sí mismo como conocedor de los principios de una sociedad de riesgo moderna. Por un lado, estas leyes de Chernóbil permitieron una organización cívica sin precedentes; por otro lado, se convirtieron en espacios de corrupción a través de los cuales se extendieron prácticas informales para proveer o vender el acceso a los privilegios y protecciones del estado (blat) ${ }^{26}$.

Informes etnográficos han ilustrado que el futuro del pos socialismo no puede basarse en modelos predictivos o tratarse como una entrada fácil en el libre mercado. Michael Burawoy y Katherine Verdery apuntaron las conexiones entre los mundos socialista y pos socialista así como la creciente dependencia entre la formación del estado pos socialista y la economía global. Dichas dependencias "han moldeado radicalmente las reglas del juego, los parámetros de acción dentro de los cuales los actores siguen sus rutinas y prácticas diarias"27. Los métodos etnográficos son críticos para elucidar estos procesos interrelacionados a nivel local. Esto es particularmente cierto en lo que se refiere a evaluar las decisiones que la gente toma ante opciones limitadas y los aspectos informales del poder que conforman dichas decisiones.

Los cambios en la condición humana agregada y las circunstancias de la ciudadanía están también en juego en estos inestables mundos político y económico. Los principios de una "ciudadanía clásica" dotan a los ciudadanos de derechos naturales y legales protegidos

\footnotetext{
24 BANCO MUNDIAL, Managing the Legacy of Chernobyl, Washington, D.C., 1994.

25 HACKING, Ian, Taming of Chance, Cambridge University Press, Cambridge, 1990.

${ }^{26}$ Para una definición del concepto de blat, ver LEDENEVA, Alena, Russia's Economy of Favours: Blat, Networking, and Informal Exchange, Cambridge University Press, Cambridge, 1998.

${ }^{27}$ BURAWOY, Michael y VERDERY, Katherine, Uncertain Transition: Ethnographies of Change in the Post socialist World, Rowman and Littlefield, Lanham, 1999, p. 2.
} 
desde el nacimiento ${ }^{28}$. Sin importar su nacionalidad, dicha protección fue garantizada para todos los habitantes de Ucrania cuando el país declaró su independencia. Aún así, los derechos de nacimiento son un garante insuficiente de protección porque las vidas de los habitantes de ciertas zonas en Ucrania no pueden ser completamente, ni siquiera parcialmente, protegidas de las amenazas medioambientales a largo plazo. Para estos habitantes, el concepto de ciudadanía en sí lleva añadida una carga de supervivencia. La adquisición y el manejo de ciertas formas democráticas relacionadas con la apertura, la libertad de expresión, y el derecho a la información son objetivos prioritarios para estar seguros. La población está todavía negociando hasta los objetivos más básicos de la protección - por ejemplo, la inclusión económica y social- usando los aspectos constitutivos de la vida. Dichas negociaciones exponen ciertos patrones reconocibles en otra cosa: el papel de la ciencia en la legitimación de las instituciones democráticas, limitando cada vez más el acceso a la sanidad y el bienestar a medida que las tendencias capitalistas tomaban el relevo, y la complicada correlación de los derechos humanos con la autopreservación biológica.

\section{Ciudadanía biológica}

En Ucrania, donde la democratización está unida a la transición a una economía de mercado, la biología herida de una población se ha convertido en la base de la pertenencia social y de las reivindicaciones de ciudadanía en juego. Las clínicas de investigación de la radiación, tanto las operadas por el gobierno como las que no, median en una economía informal de enfermedades y reclamaciones de una "ciudadanía biológica" - demanda, pero con acceso limitado, una forma de bienestar social basada en criterios médicos, científicos y legales que reconocen heridas y compensaciones por ellas-. Estas demandas son expresadas en un contexto de pérdidas de recursos primarios como el empleo y de protección estatal ante la inflación y el deterioro de la clase legal y política. Las luchas por los recursos médicos limitados y los factores que constituyen una legítima reivindicación de ciudadanía son parte de un terreno inexplorado del pos socialismo. En contra de un abrumador orden de inseguridad, hay preguntas que hacerse sobre cómo el valor de las vidas de otros es juzgado en esta nueva economía política, sobre la capacidad del conocimiento científico de empoderar políticamente a aquellos que buscaban un valor relativamente alto, y sobre los tipos de racionalidades y prácticas biomédicas que surgen respecto a nuevas indeterminaciones sociales, económicas y somáticas. La indeterminación del conocimiento científico sobre las aflicciones que enfrentaba la gente y sobre la naturaleza de la catástrofe nuclear se materializa aquí tanto como un inconveniente como una fuente de ventajas. Las ambigüedades relacionadas con la interpretación de las heridas debidas a la radiación, junto con sus inextricables relaciones con las incertidumbres sociales y políticas generadas por las intervenciones soviéticas y la actual vulnerabilidad política y económica, hacen que el alcance de la población afectada en Ucrania y de sus afirmaciones sobre el daño sufrido sea plausible, irónico y catastrófico.

Un ejemplo de cómo estas dinámicas científicas y políticas operaban en el día a día: el experto del país en lo relacionado con el desastre, Symon Lavrov, tenía buena imagen internacional por haber desarrollado modelos de efectos colaterales informatizados y calculado dosis en grandes poblaciones en el periodo pos soviético; me dijo, sin embargo, que "cuando una madre con lágrimas en los ojos viene a mi laboratorio y me pregunta, profesor Lavrov,

${ }^{28}$ SCHNAPPER, Dominique, "The European Debate on Citizenship" en Daedalus vol. 126, no 3, 1997, p. 201. 
'dígame qué le pasa a mi hijo', le asigno una dosis y no digo más. Lo duplico todo lo que puedo". La oferta de una dosis más alta aumenta la probabilidad de que la madre sea capaz de asegurarse la protección social a cuenta de su hijo potencialmente enfermo. Lavrov y la madre apenada fueron dos de las muchas figuras cuyos esfuerzos documenté. El caso es que la madre pudo ofrecer a su hijo una dosis, un lazo protector con el estado, que dependía de la probabilidad de enfermedad, un lazo biológico. Lo que ella podía ofrecer, quizá lo más preciado que podía ofrecerle a su hijo en ese contexto, era un conocimiento, historia y categoría específicos. La exposición del niño y el conocimiento que haría de dicha exposición un hecho empírico no era algo para ser reprimido o negado -como había intentado el modelo soviético- sino algo para ser convertido en un recurso y posteriormente ser distribuido por medios informales.

Los casos específicos ilustran cómo estos procesos económicos y de estado, combinados con las dinámicas técnicas ya descritas, han dejado el trabajo de base para tales "contrapolíticas"29. Los ciudadanos han llegado a depender de tecnologías y procedimientos legales alcanzables para ganarse el reconocimiento político y alguna forma de inclusión en el bienestar. Conscientes de que ellos tenían menos posibilidades de encontrar empleo y acceder a sanidad en la nueva economía de mercado, estos ciudadanos evaluaron elementos en su vida -medidas, números, síntomas- que podían estar conectados a la historia científica y burocrática de la mala gestión y el riesgo de un estado. Cuanto más estrecha era la conexión que podía establecerse, mayores las posibilidades de asegurar derechos económicos y sociales. Esta dimensión de la enfermedad como "contrapolítica" sugiere que los sufridores son conscientes de la forma en que la política moldea lo que saben y lo que no saben sobre sus enfermedades y que no les queda otra que utilizar esta política para evitar un mayor deterioro de su salud, deterioro que resultaba, en parte, de un colapso del sistema sanitario y una pérdida de las protecciones legales adecuadas.

La probabilidad en relación a las enfermedades relacionadas con la radiación se convirtió en un recurso central para la investigación científica local. Este juego con la probabilidad se estaba proyectando de nuevo en la naturaleza a través de una intrincada ciencia local, por así decirlo. Jóvenes neuropsiquiatras sacaron lo mejor de la imposibilidad de escapar de sus circunstancias políticas - no podían obtener visas para salir del país - integrando taxonomías médicas internacionales y soviéticas y desarrollando clasificaciones de desórdenes mentales y nerviosos que en literaturas expertas eran considerados muy bajos para hacer ninguna contribución biológica significativa. Por ejemplo, se involucraron neuropsiquiatras en un proyecto destinado a encontrar y atajar los casos de retraso mental en niños expuestos en el útero durante el primer año tras el desastre. En el caso de uno de estos niños, un niño cojo de nueve años, los investigadores y los padres compartieron su conocimiento para redefinir el desorden del niño y considerarlo originado por la radiación. Aunque la dosis de radiación del niño fuera baja, se le concedió el estatus de sufridor por la exposición de la madre -doctora de emergencias que eligió trabajar en la zona hasta bien avanzado su embarazo- y también porque un escáner PET reveló una lesión cerebral que nunca se trató de relacionar con otra cosa que no fuera la radiación - podía haber sido un trauma de nacimiento-. Mientras los

${ }^{29}$ GORDON, Colin, "Government Rationality: An Introduction" en BURCHELL, Graham; GORDON, Colin y MILLER, Peter (eds.), The Foucault Effect: Studies in Governmentality, The University of Chicago Press, Chicago, 1991, p. 5. 
investigadores construían una muestra humana para la investigación, también estaban construyendo el destino de los nuevos sujetos humanos de investigación. Era precisamente ese destino el que los padres querían ofrecer a sus hijos - una ciudadanía biológica.

Estas afirmaciones y prácticas relacionadas con la radiación constituyeron una forma de trabajo en la transición a una economía de mercado. Un administrador coincidía en que las reclamaciones por enfermedades de radiación entre la población ucraniana equivalían a una forma de "compensación de mercado". Me dijo,

"si la gente pudiera mejorar el presupuesto familiar, habría muchas menos enfermedades. Ahora la gente está orientada hacia una cosa. Creen que sólo a través de las enfermedades, particularmente las complicadas o incurables pueden mejorar los presupuestos familiares".

Los administradores como él me informaron de que no debía "culparles con demasiada dureza" por contribuir a una economía informal de diagnósticos y subsidios. Las complicidades se podían encontrar a todos los niveles, y los conflictos morales que entrañaban eran discutidos públicamente. Otro administrador que autentificaba reclamaciones de compensaciones me dijo que las enfermedades se habían convertido en una especie de divisa. "Hay mucha gente que no trabaja," dijo. "La gente no tiene dinero para comer. El estado ya no da medicinas gratis. Las farmacias son comercializadas". Para él, este trabajo era como el de un banco: "El diagnóstico que escribimos es dinero".

La historia de Anton y Halia - de cuarenta y dos años en 1997- muestra cómo funcionaba dicha complicidad en el terreno más personal. Las nuevas instituciones, procedimientos y actores que trabajaban para el estado, en la clínica de investigación o para organizaciones cívicas llegaban hasta el Kvartyra - apartamento- de la pareja. La identidad de trabajador de Anton, su sentido de la masculinidad, y su papel de padre y cabeza de familia, estaban siendo violentamente dislocados y alterados en el proceso. En 1986, el estado reclutó a Anton para trabajar seis meses en la Zona de Exclusión, transportando bolsas de óxido, arena y grava hasta el reactor. Las bolsas eran trasladadas y depositadas usando helicópteros. Él no tenía ni idea de cuanta radiación absorbió durante esos seis meses. De 1991 en adelante, Anton pasaba rutinariamente por el sistema clínico, monitoreado como cualquier otro "posible" inválido. Sus síntomas aumentaron con el tiempo. Tenía dolores de cabeza crónicos, perdía la memoria a corto plazo, exhibía un comportamiento antisocial, desarrolló un trastorno en el habla, y tenía convulsiones e impotencia, así como otros muchos problemas. A pesar de sus crecientes y cada vez más intensos síntomas, su diagnóstico no "pasó" de la lista inicial como caso "psicosocial".

Cuando conocí a Anton y a su mujer, Halia, intentaban salir adelante con la pequeña pensión que él recibía como sufridor. Anton se vio en bancarrota tanto moral como económicamente:

"el estado me quitó la vida. Me la arrancó, se fue. ¿Qué hay de feliz en eso? Un hombre honorable no puede sobrevivir ahora. ¿Para qué? ¿Para qué? Teníamos una vida. Teníamos mantequilla. Teníamos leche. No puedo comprar una plancha. Antes podía comprar cincuenta planchas. El dinero estaba ahí. El salario de mi mujer es menor de lo que cuesta una plancha". 
Me dijo que no sabía cómo comerciar con bienes o venderlos en el mercado. Su pensión dejó a Anton con muy pocas opciones. Se encontró enfrentando la vergonzosa elección entre ganarse el pan con su enfermedad en el sistema de compensaciones de Chernóbil o la pobreza. Con el tiempo, en un esfuerzo coordinado para modificar la etiqueta psicosocial de Anton, la pareja coincidió con el líder de un grupo activista de trabajadores discapacitados en una clínica. A través de él conocieron a un neurólogo que conocía al director del comité de trabajo médico local. La pareja esperaba que este individuo proporcionara a Anton la ayuda oficial que reclamaba por su discapacidad debida a Chernóbil.

La motivación económica de estas acciones estaba clara. Aún así, era difícil para mí ver a este hombre dejando de lado todo lo que sabía o pensaba sobre sí mismo para probar que sus difusos síntomas tenían una base orgánica. La neurología fue una puerta clave hacia la discapacidad; los desórdenes neurológicos eran más ambiguos pero más fáciles de probar usando tecnologías de diagnóstico, auto-inducciones y exhibiciones corporales. A cada paso, Anton se rompía mentalmente, cayó en un patrón de comportamiento agresivo. Su apuesta médico-legal -el ganarse la vida en esta nueva economía de mercado gracias a la enfermedad - reflejaba las prácticas de toda una ciudadanía falta de ingresos o de medios para generarlos. Este enfoque se ha convertido en sentido común, o en palabras de Clifford Geertz, lo que "queda cuando toda clase de sistemas simbólicos articulados han agotado sus deberes" ${ }^{\prime \prime 3}$.

Cuando volví en el 2000 a Kiev para una investigación más amplia, descubrí que los actuales políticos demócratas, muchos de los cuales redactaron las leyes originales de compensación de nacionalistas y soberanistas, ahora veían el sistema de compensaciones de Chernóbil como un desgraciado error que "accidentalmente" reprodujo una población como la socialista. Los fondos y los grupos activistas eran sustentados por los líderes socialistas y comunistas, que ejercían presión para que continuaran las ayudas en un parlamento cada vez más dividido. Mientras tanto, las agencias internacionales como el Banco Mundial se referían al aparato social de Chernóbil como un "peso muerto" para la, menos que ideal, transición de Ucrania a una economía de mercado. Los banqueros estaban tan predispuestos contra el sistema que pusieron como condición para conceder futuros préstamos una rápida liquidación del mismo. La desaparición de esta población expuesta del radar del estado parece cada vez más probable. Una vez "protegida" por un estado preocupado por la seguridad, esta población expuesta está siendo abandonada a sus síntomas y desarraigo social.

Las opiniones sobre cómo debería el estado abordar el destino de estas víctimas de Chernóbil sirven también como una especie de barómetro del cambio moral en el país. Los habitantes del medio rural que normalmente eran los que menos recibían en las redistribuciones socialistas tienden a simpatizar con la lucha de las víctimas. Entre los habitantes de Kiev y otros centros urbanos, hay un creciente consenso en que los inválidos son "parásitos del estado que dañan la economía y no pagan impuestos". Muchos jóvenes que habían sido evacuados de la zona no quieren que se les relacione con grupos de sufridores porque esto les dificulta encontrar empleo.

${ }^{30}$ GEERTZ, Clifford, Local Knowledge: Further..., op. cit. 
Chernóbil fue un evento político clave que tuvo múltiples efectos, algunos de los cuales están aún por descubrir, la verdad sólo se ha hecho saber en parte a través de estimaciones derivadas de la ciencia experimental. El discurso inmediato de la posindependencia en Ucrania se centró en la "verdad" sobre Chernóbil. Los ucranianos intentaron poner su sufrimiento en perspectiva frente al modelo represivo de ciencia y estado: el número de personas que murió, cómo el gobierno engañó a los ciudadanos sobre la escala del desastre, cómo los mapas de contaminación no se correspondían con la realidad etc. Mientras la dura realidad de los mercados se metía en la vida cotidiana, este modelo de organizar el sufrimiento dejó espacio rápidamente a diferentes tipos de negociación científica y política, que tenía que ver directamente con el mantenimiento, y de hecho la nueva creación, de un estado y una población pos socialista.

$\mathrm{Si}$, al nivel del estado moderno, las esferas políticas y de producción científica están en un proceso permanente de intercambio y estabilización mutua, entonces lo que he sugerido aquí es que la estabilización demostró ser una tarea mucho más complicada. En la catástrofe de Chernóbil estaba en juego un peculiar campo de poder pos socialista en proceso de construcción que usaba la ciencia y las categorías científicas para establecer el alcance del estado. Científicos y víctimas también están estableciendo sus propios conocimientos relacionados con las heridas como medio de negociar reconocimiento público, poder político y más protección del estado en forma de compensación económica y cuidados médicos. La biología se convierte en un recurso en sentido multidimensional - una materia versátil que pueden hacer aparecer un estado y nuevas poblaciones-. Este campo de poder pos socialista va acompañado de unos aspectos físicos, experimentales, políticos, económicos y espaciales específicos. Va sobre conocimiento e ignorancia construida, visibilidad e invisibilidad, inclusión y exclusión, probabilidades y hechos, y la división de protección y bienestar que no encajaban con los modelos predecibles. También va de cómo individuos y poblaciones pasan a formar parte de nuevos regímenes de cooperación en investigación científica y en formas locales de protección humana promovidas por el estado. En este contexto, el sufrimiento se obtiene y materializa en sus dimensiones legal, económica y política. A la vez, estas materializaciones constituyen un sentido común representado por los sufridores que puede promover la protección e intensificar nuevos tipos de vulnerabilidad en las esferas doméstica, científica y burocrática.

\section{Bibliografía}

BANCO MUNDIAL, Managing the Legacy of Chernobyl, Washington, D.C., 1994.

BECK, Ulrich, "The Anthropological Shock: Chernobyl and the Contours of a Risk Society", en Berkeley Journal of Sociology, vol. 32, 1987, pp. 153-165.

BURAWOY, Michael y VERDERY, Katherine, Uncertain Transition: Ethnographies of Change in the Post socialist World, Rowman and Littlefield, Lanham, 1999.

DAS, Veena, Critical Events: An Anthropological Perspective on Contemporary India, Oxford University Press, Nueva Deli, 1995.

DUMIT, Joseph, Picturing Personhood: Brain Scans and Biomedical Identity, Princeton University Press, Princeton, 2004.

FARMER, Paul, Infections and Inequalities: The Modern Plagues, University of California Press, Berkeley, 1999. 
GEERTZ, Clifford, Local Knowledge: Further Essays in Interpretive Anthropology, Basic Books, Nueva York, 1983.

GORDON, Colin, "Government Rationality: An Introduction" en Burchell, Graham, Gordon, Colin y Peter Miller (eds.), The Foucault Effect: Studies in Governmentality, The University of Chicago Press, Chicago, 1991, pp. 1-51.

GRAHAM, Loren, What Have We Learned about Science and Technology from the Russian Experience?, Stanford University Press, Stanford, 1998.

HACKING, Ian, Taming of Chance, Cambridge University Press, Cambridge, 1990.

HIPPEL, Frank von, Citizen Scientist, The American Institute of Physics, Nueva York, 1991.

KHARKHORDIN, Oleg, The Collective and the Individual in Russia: A Study of Practices, University of California Press, Berkeley, 1999.

KLEINMAN, Arthur y KLEINMAN, Joan, "The Appeal of Experience; The Dismay of Images: Cultural Appropriations of Suffering in Our Times" en Daedalus, vol. 125, $\mathrm{n}^{\circ} 1,1999, \mathrm{pp} .1-24$.

KLEINMAN, Arthur, Social Origins of Distress and Disease, Yale University Press, New Haven, 1986.

LEDENEVA, Alena, Russia's Economy of Favours: Blat, Networking, and Informal Exchange, Cambridge University Press, Cambridge, 1998.

MARTIN, Emily, Flexible Bodies: Tracking Immunity in American Culture from the Days of Polio to the Age of AIDS, Beacon Press, Boston, 1994.

PETRYNA, Adriana, Life Exposed: Biological Citizens After Chernobyl, Princeton University Press, Princeton, 2002.

PROCTOR, Robert, Cancer Wars: How Politics Shapes What We Know and Don't Know about Cancer, Basic Books, Nueva York, 1995.

RAPP, Rayna, Testing Women, Testing the Fetus: The Social Impact of Amniocentesis on America, Routledge, Nueva York, 1999.

SCHNAPPER, Dominique, "The European Debate on Citizenship" en Daedalus vol. 126, n. 3, 1997.

SHCHERBAK, Yurii, "Ten Years of the Chernobyl Era", en Scientific American, abril, 1996.

$\mathrm{SICH}$, Alexander, "The Denial Syndrome (Efforts to Smother the Burning Nuclear Core at the Chernobyl Power Plant in 1986 Were Insufficient)" en Bulletin of Atomic Scientists, vol. 52, 1996, pp. 3840. 


\section{RELACIONES INTERNACIONALES}

Revista académica cuatrimestral de publicación electrónica Grupo de Estudios de Relaciones Internacionales (GERI) Universidad Autónoma de Madrid, España

www.relacionesinternacionales.info

ISSN 1699 - 3950

ff facebook.com/RelacionesInternacionales

twitter.com/RRInternacional 